\title{
ORIGINAL
}

\section{PERFIL DE LA DEMANDA URGENTE E INFLUENCIA DEL FÚTBOL TELEVISADO EN UN SERVICIO EXTRAHOSPITALARIO EN LA ZONA BÁSICA DE SALUD DE TAFALLA, NAVARRA}

Ignacio Pérez-Ciordia (1), Francisca Catalán Fabo (1), Fernando Zalacain Nicolay (1), Maite Barriendo Antoñanzas (1), Ramón Solaegui Diaz de Guereñu (1) y Francisco Guillén Grima (2)

(1) Centro de Salud de Tafalla. Navarra.

(2) Departamento Ciencias de la Salud. Universidad Pública de Navarra.

\section{RESUMEN}

Fundamento: La demanda a los servicios de urgencias, tanto hospitalarios como extrahospitalarios, mantiene un ritmo creciente. El objetivo del presente trabajo es doble: cuantificación y estudio las características personales de los usuarios demandantes de atención urgente y valorar si el fútbol televisado influye en la utilización del servicio de urgencia.

Métodos: Estudio descriptivo longitudinal (9.723 usuario demandantes) y estudio de casos y controles (1.284 usuarios demande regresión logística La o no de fútbol televisado mediante modiante la odds ratio (OR). Se excluyen del estudio las consultas telefónicas y las de enfermería

Resultados: El 10,6\% de la demanda ha correspondido a consulas en domicilio y se han remitido al hospital el $4,8 \%$ del total. E $13,3 \%$ de la demanda corresponde a personas desplazadas de otras zonas de salud y son 65 los usuarios hiperfrecuentadores con 8 o más consultas. El mes de agosto (32,3\%), el domingo (44,56\%) y el grupo horario de 12 a 14 horas $(8,38 \%)$ son los momento de mayor demanda de atención, siendo las diferencias estadísticamente significativas. El fútbol televisado se asoció a un aum
del $19,8 \%$ (p<0,001) respecto al periodo control.

Conclusiones: Se observa una alta frecuentación a los servicios de urgencia extrahospitalaria, con una importante concentración de ción prestada a personas desplazadas. La retransmisión de fútbol televisado se asocia con una mayor utilización del servicio de urgencias.

Palabras clave: Atención Primaria de salud. Servicios médicos de urgencia. Necesidades y demanda de servicios de salud. Fútbol.

Correspondencia:

Pérez-Ciordia Ignacio

C/ Rio Ega $232^{\circ} \mathrm{C}$ Pamplona (Navarra)

Correo electrónico: dicastillo01@ terra.es

Teléfono: 948236584

\section{ABSTRACT}

Profile of the Emergency Demand and Influence of Televised Soccer Games on an Extrahospital Center in the Tafalla Healthcare District. Navarre, Spain

Background: The demand placed on both hospital as well as extrahospital emergency care units currently continues to increas at a growing rate. This study has a twofold objective: the quantification and study of the personal characteristics of the users who are demanding emergency care and assessing whether televised soccer ices.

Methods: A longitudinal descriptive study ( 9,723 users demanding care) and study of cases and controls (1,284 users demanding care) according to whether or not a soccer game was being televise by means of a logistic regression model. The associations were quantified by means of the odds ratio $(\mathrm{OR})$. Those dealt with by telepho-
ne or in infirmaries were not included in the study.

Results: A total $10.6 \%$ of the demand involved home visits, $4.8 \%$ of this total having been sent to hospital. A total $13.3 \%$ of the demand corresponds to individuals visiting from other healthcare districts, a total of 65 being overusers of emergency care ( 8 or more visits). The month of August (32.3\%), Sundays (44.56\%) and the 12:00 p.m.-2:00 p.m. timeframe (8.38\%) are the times when the greatest demand for care occurs, the differences being statistically significant. Televised soccer games were associated to a $19.8 \%$ (p

Conclusions: A high degree of use of the extrahospital emergency care units has been found to exist, a major part of the deman being concentrated at highly specific points in time. A major degre of care is provided to those visiting from outside their own healthcate districts. Television soccer game broadcasts is associated with the greater utilization of the emergency care services.

Key words: Primary health care. Emergency medical services. Health services needs and demand. Football. 


\section{INTRODUCCIÓN}

La atención sanitaria a las urgencias es uno de los elementos que mejor definen y caracterizan los servicios sanitarios de una comunidad $^{1,2}$. La demanda urgente de asistencia sanitaria se ha incrementado en los últimos años, tanto en atención primaria como en el sector hospitalario ${ }^{3,4}$, oscilando entre un $13,3 \%$ y un $4,4 \%$ respectivamente de crecimiento vegetativo anual, a pesar de que se pensó que disminuiría ${ }^{5}$ con la Reforma de la Atención Primaria. La definición oficial de urgencia es propuesta por la Asociación Médica Americana y suscrita por la Organización Mundial de la Salud ${ }^{7}$. No obstante, el interesado interpreta de manera muy diferente la necesidad de asistencia urgen$\mathrm{te}^{2,8}$.

Se ha calculado que en nuestro país en el año 2000 treinta millones de ciudadanos demandaron asistencia urgente en el ámbito hospitalario y extrahospitalario ${ }^{6}$. Para Navarra, la demanda de asistencia urgente extrahospitalaria ha pasado de 46.425 en el año 1992 a 93.811 en el año 2000. En la zona básica de Tafalla (ZBT), la demanda ha sido de 6.672 y de 10.285 pacientes, respectivamente, con un crecimiento constante y progresivo (Memorias de Servicio Navarro de Salud).

El conocer las características clínico-epidemiológicas que presenta la población atendida en nuestra zona de salud, supone un primer acercamiento a conocer las necesidades reales y a la instauración de medidas que faciliten el proceso de atención.

La facilidad de acceso por parte de la población a los servicios de urgencias, provoca que éstos se encuentren sometidos a fluctuaciones periódicas. Estos cambios dependen mas de factores sociales o coyunturales que de factores específicos de la propia enfermedad. Así, se ha relacionado la demanda de atención urgente con aspectos tales como la adecuación a los horarios labo- rales y escolares ${ }^{9,10}$ y con la retransmisión por televisión de actividades deportivas ${ }^{11}$.

El objetivo del estudio es realizar un análisis descriptivo de la demanda urgente de nuestra zona y relacionar la retransmisión de fútbol televisado con la solicitud de demanda de atención urgente mediante análisis de periodo-control.

\section{SUJETOS Y MÉTODOS}

El periodo de estudio comprende de 1 de febrero de 2001 a 1 de febrero de 2002 en la ZBT que tiene una población de 13.124 habitantes (fuente: Tarjeta de Identificación Sanitaria-TIS- a fecha 15 de junio de 2001) distribuida en 20 núcleos de población. Comprende Tafalla y Otras Poblaciones (OP) entendiendo como tal el resto de poblaciones, que completan la zona básica y situados a una media de 12 Kilómetros del centro de salud.

Para cubrir la demanda en horario de mañana ( 8 a 15 horas) existe un centro de salud en la localidad principal Tafalla, atendido por 6 médicos de atención primaria y 2 pediatras, y varios consultorios locales. El hospital más cercano se encuentra en la capital de la provincia a media hora por carretera de la localidad principal. El servicio de atención de urgencias, ubicado en el propio centro de salud, es atendido por 4 médicos con una dependencia orgánica y funcional diferente y ajena al equipo de atención primaria. El horario de trabajo es desde las 15 horas a las 8 horas del día siguiente en días laborales y 24 horas los festivos. En todo el tramo horario, la guardia es de presencia física y el equipo de guardia está integrado por 1 médico, 1 Diplomado en enfermería (DUE) y 1 celador. La dotación de material clínico es adecuada $^{2}$.

Definimos como urgencia toda demanda de asistencia médica en horario de trabajo propio del servicio de urgencias, incluyendo

Rev Esp Salud Pública 2003, Vol. 77, N. ${ }^{\circ} 6$ 
tanto la realizada en el centro de salud como en los domicilios; se incluye también la asistencia realizada en accidentes de tráfico, con desplazamiento al lugar del mismo. No se incluyen las consultas telefónicas ni la demanda de asistencia exclusiva de los DUE.

Persona desplazada es aquella demandante de asistencia con su médico de cabecera en otra zona de salud diferente e incluye también a los que no tienen médico oficialmente asignado.

Se define como hiperfrecuentador al usuario que demanda 8 o más consultas (percentil 99), estudiandose en este trabajo sus características especificas como grupo.

La edad está referida a la edad de cada individuo en su primer episodio de atención.

En un cuestionario elaborado a propósito, se recogió información sobre fecha y hora de la solicitud de la demanda de asistencia, nombre y apellidos del enfermo, sexo y edad, médico de cabecera asignado, atención en centro de salud o en domicilio y datos del médico que realizó la asistencia. Así mismo se recogieron datos sobre el diagnóstico realizado, el cual se codifica según la Clasificación Internacional de Problemas de Salud en Atención Primaria CIPSAP-2 Definida (Wonca) ${ }^{12}$ y remisión, si procede, a centro hospitalario.

El centro de salud está informatizado con el programa Omni-AP (3.0) con fácil acceso para el equipo de urgencias, el cual proporciona información tanto de datos administrativos como de antecedentes previos de los usuarios adscritos a la zona de salud.

Los códigos de la CIPSAP-2-Definida se agrupan para su estudio en 18 secciones, adaptadas a la Clasificación Internacional de Enfermedades (CIE). Algunas demandas de la población no vienen codificadas; se toma la decisión de codificar con categoría propia estos problemas. Son básicamente los accidentes de trafico y las defunciones.

Para el mismo periodo de tiempo se recogieron datos de la retransmisión de partidos de fútbol (Liga de $1^{\circ}$ División y UEFA): hora de inicio y final del partido (entendido como hora de inicio de la retransmisión deportiva hasta hora de inicio del siguiente programa televisivo), y tipo de campeonato. La información se obtuvo de un periódico provincial en el apartado de Televisión. La recogida de información abarca las seis televisiones gratuitas que se visualizan en Navarra. Se recogió la misma información referida a la retransmisión en modalidad gratuita o pago por visión del único equipo de fútbol de primera división de Navarra.

El registro de la información ha sido realizado por los autores. Los diagnósticos WONCA no se han recogido cuando han sido profesionales diferentes los que han realizado el diagnostico.

Se consideró como caso a la persona demandante de asistencia urgente en horario de retransmisión de la actividad deportiva y como control al usuario que demandó asistencia sanitaria en las 2 horas anteriores o posteriores a la retransmisión del fútbol televisado.

El análisis estadístico de los datos se realizó mediante los programas Epiinfo.6 y SPSS.10. Las variables cuantitativas se resumieron como medias (DE) y se compararon mediante el análisis de la varianza (ANOVA). Las variables cualitativas se comparan mediante el test de la ji-cuadrado de Pearson y las asociaciones se han cuantificado mediante la odds ratio (OR). Se aceptan que las diferencias son estadísticamente significativas cuando el valor de p es inferior a 0,05 o cuando el Intervalo de confianza (IC) de las diferencias de porcentajes no incluye el 0 . Se realizó análisis multivariante mediante regresión logística para analizar la asociación entre demanda de consulta en horario 
de retransmisión de fútbol televisado y demanda fuera de la retransmisión de dicha actividad.

\section{RESULTADOS}

En el periodo de estudio se atendió un total de 9.723 consultas, lo que representa una media de 35,5+/-24 consultas/día (rango: 9-73). Del total de consultas el 10,6\% (1.027) fue visita a domicilio y 466 casos $(4,8 \%)$ fueron remitidos al hospital. A la ZBT correspondieron 8.426 consultas $(86,7 \%)$, con una demanda de $722 / 1000$ habitantes / año.

Respecto al día de la semana (tabla 1), el domingo fue el que mayor porcentaje de urgencias se atendieron $(22,7 \%)$, siendo las diferencias estadísticamente significativas $\left(\mathrm{F}_{6,360,0.000}=48,939\right)$. El 44,9\% de la variabilidad de la demanda es explicada por la variable día $\left(\mathrm{eta}^{2}=0,449\right)$.

En la distribución por mes (tabla 2), se observaron diferencias significativas $\left(\mathrm{F}_{11,355,0.006}=2,453\right)$ y el $7,1 \%$ de la variabilidad de la demanda $\left(\mathrm{eta}^{2}=0,071\right)$ es explicada por la variable mes. Los meses de enero y agosto comprenden el $20,1 \%$ del total de la demanda.

En la distribución por grupo horario (tabla 3 ) en el que se solicitó la atención se observaron diferencias estadísticamente significativas con $\mathrm{F}_{7,2340,0000}=570,107 \mathrm{y}$ el $63 \%$ de la variabilidad $\left(\mathrm{eta}^{2}=0,630\right)$ es explicada por la variable hora. El 48,9\% del total de la demanda de urgencias se solicitó en el periodo de 18 a 23 horas.

La edad media de los pacientes fue de $35,5+/-24,1$ años con un rango de 0,5 a 100 años. El grupo porcentualmente mas demandante fueron los menores de 5 años y dentro de este grupo el 13\% correspondió a menores de 1 año. La frecuencia de demanda disminuyó con la edad, excepto para el grupo
Tabla 1

Distribución de las consultas por día de la semana. ANOVA. Zona básica de Tafalla

\begin{tabular}{|c|c|c|c|}
\hline DIA & MKIDIA & $\mathbf{N}$ & os \\
\hline Douningo & 41.56 & 52 & 9.25 \\
\hline I uncsi & 24.56 & 52 & $10,0,4$ \\
\hline Marie: & 22.77 & 52 & 5,79 \\
\hline Miérooles & 21.58 & 52 & 7,14 \\
\hline Jucres & 23,38 & 53 & 7.25 \\
\hline Vicuncs & 25.12 & 53 & 9,24 \\
\hline Sishotde & 23.4 & 53 & $7,6 \mathrm{x}$ \\
\hline \multirow[t]{2}{*}{ Total } & 26,19 & 367 & 11.1 \\
\hline & $00,0.000^{-18}$ & & \\
\hline
\end{tabular}

DS: desviación estandar

Tabla 2

Distribución de las consultas por mes.

ANOVA. Zona básica de Tafalla

\begin{tabular}{|c|c|c|c|}
\hline Mes & Media & $\mathrm{N}$ & US \\
\hline linem & 3) 4 , & 31 & 15,1 \\
\hline Febreso & 21,63 & 30 & 8,66 \\
\hline Manzo & 25.74 & 31 & 8.18 \\
\hline Nhnl & 29,37 & (4) & 13,2 \\
\hline Manys & 27,75 & 31 & 10) 58 \\
\hline Junio & 25,87 & 30 & 897 \\
\hline Julio & 26,13 & 31 & 8.98 \\
\hline Apwsts & 32,32 & 31 & 13,38 \\
\hline Sepliemhre & 24,67 & 3) & $9 \& 8$ \\
\hline Octubre & 21,65 & 31 & 10,50 \\
\hline Novismbe & 22.77 & $3)$ & 8.26 \\
\hline Ihacanhtre & 26,68 & 31 & 11,78 \\
\hline \multicolumn{4}{|c|}{$F_{11,398,000}-2.453$} \\
\hline
\end{tabular}

DS: desviación estandars

Rev Esp Salud Pública 2003, Vol. 77, N. o 
Tabla 3

Distribución de las consultas por grupo horario. ANOVA. Zona básica de Tafalla.

\begin{tabular}{|c|c|c|c|}
\hline HORA & MEDIA & $\mathbf{N}$ & DS \\
\hline $15-17$ & 6.65 & $36 x$ & 2.556 \\
\hline 1820 & $8.2 \mathrm{k}$ & 366 & 3,138 \\
\hline $21-2.7$ & 47 & 366, & $2.3 \times 9$ \\
\hline $0-2$ & 1,30 & 366 & 1,307 \\
\hline $3-5$ & $0 . \%$ & $36 x^{\prime}$ & $1.08 \times$ \\
\hline 68 & 0,93 & 366 & $1,19 / 4$ \\
\hline $4-11$ & 97 & 76 & 5559 \\
\hline $12-14$ & $8,3 k$ & 76 & 463 \\
\hline \multirow[t]{2}{*}{ Thinal } & 4,14 & 2.748 & 3.972 \\
\hline & andoos -5 & & \\
\hline
\end{tabular}

DS: desviación estandar

de 20 a 29 años en el que se mantuvieron cifras altas (tabla 4). Para los mayores de 65 años se observaron diferencias estadísticamente significativas $(\mathrm{p}=0,000)$ en la remisión a hospital y en la atención en el domicilio $(\mathrm{p}=0,000)$. Para la población censada en los municipios adscritos a la zona básica los menores de 5 años presentaron una tasa de 172 por 100 habitantes, descendiendo progresivamente a partir de esta edad.

No se observaron diferencias por sexo. Las mujeres demandaron mas atención a domicilio (OR: 1,52; IC:1,33-1,74) que los hombres, mientras que éstos son derivados al hospital con más frecuencia que las mujeres (OR:1,36; IC:1,12-1,64).

Para la población adscrita a la ZBT, los usuarios demandantes de OP se remitieron más a hospital (OR:1,85; IC:1,26-2,71) y se realizaron mas visitas a domicilio (OR:1,37; IC:1,04-1,79) que en los demandantes de Tafalla. Así mismo, los pacientes vistos en su domicilio se remitieron más al hospital que los visitados en consulta (OR:4,22; IC:3,41-5,22).

La clasificación de los diagnósticos por códigos Wonca para todos los episodios se observa en la tabla 5. Los grupos XVII, XVI y VIII comprenden el 53,9 \% del total de casos diagnosticados.

Del total de pacientes adscritos a la zona de salud, la frecuencia osciló entre 1 y 48 consultas. La edad media de los hiperfrecuentadores es de 37,3+/-27,2 años con un rango entre 0,5 y 89 años.

Hubo un total de 65 personas hiperfrecuentadoras, de las cuales 33 son hombres y 32 son mujeres. Entre todos ellos han generado un total de 833 consultas $(8,6 \%)$ con 206 visitas a domicilio (20\%) y 20 hospitalizaciones $(4,3 \%)$ sobre el total de consultas.

Sobre un total de 1.285 usuarios demandantes se observó un aumento significativo de la demanda durante la retransmisión televisada de fútbol respecto a las horas en que no hubo fútbol televisado $(19,8 \% ; \mathrm{p}<0,001)$. En el análisis multivariante (tabla 6) no se observaron diferencias estadísticamente significativas respecto a la derivación al hospital, atención domiciliaria o en CS y atención a usuario de la ZBT o a desplazados, mientras que el sexo, edad, horario, tipo de campeonato, retransmisión por televisión y diagnóstico Wonca, fueron predictores independientes de la demanda de urgencias con fútbol televisado. Así, los hombres tuvieron una probabilidad de demandar una urgencia, siempre tras ajustar por las otras variables incluidas en el modelo, un $25 \%$ menor que las mujeres (OR: 0,75$)$ y los pacientes de 25 a 29 años y de 50 a 54 años algo menos de la mitad de riesgo de demandar una atención urgente respecto al grupo etario de 30 a 49 años. En todos los grupos horarios se observó un descenso muy importante en la solicitud de demanda respecto al horario de 21 a 23 horas. Cuando la retransmisión deportiva se realizó a través de una televisión pública 
Tabla 4

Porcentaje de consultas, visitas a domicilio y hospitalización por grupo de edad. Zona básica de Tafalla

\begin{tabular}{|c|c|c|c|c|}
\hline Gruponedad & Tasa* 10000 & $\%$ Cinsultas & $\%_{v}$ Dun $/$ Cous & $\%$ Hosp ${ }^{\prime}$ Cins \\
\hline 0.4 & 17.2 & 11,2 & 0.3 & 2,8 \\
\hline $9-9$ & 82 & 5,7 & 07 & 3,4 \\
\hline $10-14$ & 8.4 & 4.7 & 0.7 & 3,1 \\
\hline $15-19$ & 10.1 & 6.2 & 3.2 & 1.6 \\
\hline 20124 & 7,8 & 9,2 & 2.1 & 4,7 \\
\hline $2.7-2.9$ & 74 & 9,7 & 3,1 & 3,7 \\
\hline $30-31$ & 6 & 8 & 3.9 & 4 \\
\hline $35-39$ & 6.2 & 3.9 & 8.4 & 2.4 \\
\hline $40 ! 44$ & 4,4 & 5,1 & 3,9 & 3,5 \\
\hline $47-49$ & 4,5 & 44 & 6,9 & 5 \\
\hline $50-51$ & 5 & 4.5 & 11,8 & 3,9 \\
\hline $55-59$ & 5 & 3.8 & 9.3 & 5.7 \\
\hline 60164 & 4,5 & 2.8 & 10,3 & 7,7 \\
\hline $67-9,19$ & 5,7 & 42 & 222 & 6,1 \\
\hline $70-74$ & 5.5 & 4.1 & 369 & 9.9 \\
\hline $75-79$ & 5.2 & 3.3 & 36.8 & 7.1 \\
\hline Nol 84 & 4,9 & 2.3 & 59,6 & 9,2 \\
\hline$\times 5-89$ & 5.8 & 2 & $6 \$ 9$ & 11.9 \\
\hline $20-94$ & 2.7 & 0.7 & 72,1 & 14.7 \\
\hline $25 y(+)$ & 4.1 & 0.1 & 80 & 10 \\
\hline Total & 6.1 & 100 & 10,5 & 4.7 \\
\hline
\end{tabular}

(OR:0,29) la probabilidad de acudir a urgencias es casi tres veces menor que con la retransmisión en una televisión privada y cuando hay una doble retransmisión en televisión pública y privada, la probabilidad es un $60 \%$ menos que en televisión privada. $\mathrm{La}$ retransmisión del campeonato de la UEFA (OR:1,94) se presentó como un factor directamente asociado con la solicitud de demanda.

\section{DISCUSIÓN}

A la hora de realizar comparaciones entre nuestro trabajo y el de otros autores, hay que tener en cuenta las diferentes situaciones en que se presta el servicio de urgencias (EAP,PAC, SNU, horarios, situación geográfica, distancia al hospital.....) y el hecho de que solamente se recogen datos de asistencia exclusivamente realizada por el médi-

Rev Esp Salud Pública 2003, Vol. 77, N. ${ }^{\circ} 6$ 
PERFIL DE LA DEMANDA URGENTE E INFLUENCIA DE FUTBOL TELEVISADO EN UN SERVICIO.

Tabla 5

Distribución de los pacientes según diagnóstico WONCA. Zona básica de Tafalla

\begin{tabular}{|c|c|c|c|c|}
\hline Grupo WONCA & Cinligu & $\%$ Cinusultas & $\%$ Dum/Cous & $\%$ Heop/Cons \\
\hline Infecciosns & I & 2.57 & 6,78 & 2,12 \\
\hline Nouplasiass & II & 0.27 & 68 & 4 \\
\hline | & III & 0.35 & 31.2 .5 & 3,12 \\
\hline De la sunpere & IV & 007 & 0 & 0 \\
\hline Meatales & $\mathrm{V}$ & 3,38 & 36,01 & 5,14 \\
\hline I $\mathrm{c}$ lus suntidus & VI & 9.08 & 5.15 & 1.92 \\
\hline Carculakions, & VII & 2.59 & 2.5 & 20,9 \\
\hline Respinaksrio & VIII & 18,35 & 5,57 & 201 \\
\hline Dipestivo & IX & 62 & 11.05 & 5,61 \\
\hline Genitouina is & $\mathrm{x}$ & $2 . X$ & 9,93 & 331 \\
\hline 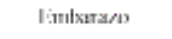 & $\mathrm{XI}$ & 0.27 & $\theta$ & is \\
\hline Piel & XII & 356 & 1,49 & 1,19 \\
\hline Loconsotor & XIII & 8,05 & 11,35 & 1,49 \\
\hline Congeriitax & XIV & 0.02 & 0 & 0 \\
\hline Pemialial & $x V$ & 0 & a & 0 \\
\hline Mul definulos: & XVI & 15,45 & 20,49 & 584 \\
\hline Lesiones & XVII & 20,12 & 2,7 & 698 \\
\hline Suplanxuta ia & XVIII & 6.05 & 5.4 & 1.8 \\
\hline 今e Trialico & 100 & 0,46 & $42 . \mathrm{K} 6$ & 47,62 \\
\hline Miserie: & 111 & 0,34 & 100 & $n$ \\
\hline Tatal & & $1(x)$ & $10.2 y$ & $4,6.3$ \\
\hline
\end{tabular}

$\%$ Dom/Cons: Porcentaje de visitas domiciliarias entre episodios vistos para cada grupo

$\%$ Hosp/Cons: Porcentaje de remisión a hospital entre episodios vistos para cada grupo

co. Además, la distribución de edad en la población condiciona la comparación de nuestros datos con otros estudios que no se basen en poblaciones similares. Todo ello hace que los resultados no puedan ser totalmente comparables con otros estudios.

El año abarcado en el periodo de estudio fue típico en cuanto a la situación epidemiológica, no existiendo ninguna situación epidémica que condicionara el tipo y volumen de demanda de urgencias.

A la vista de los resultados, nuestra zona presenta una alta tasa de demanda de aten- ción médica urgente (722/1.000 habitantes/año) $)^{2}$.

La mayor demanda de consulta en domingo respecto a los días laborables puede ser explicada por una mayor amplitud horaria, y por la ausencia de consulta medica en días festivos. Si se elimina el domingo de los días de la semana, las diferencias observadas continúas siendo significativas. Los sábados y domingos comprenden el $40 \%$ de derivación al hospital y el $38,5 \%$ del total de la atención domiciliaria.

Los meses de enero y agosto comprenden el $20,1 \%$ del total de la demanda urgente. En 
Tabla 6

Demanda de urgencias según características de los pacientes y entorno con fútbol televisado. Regresión logística.

\begin{tabular}{|c|c|c|c|c|c|c|}
\hline Factor & $\mathbf{N}$ & $\%$ & ORl, & IC $95 \%$ & Ora & IC $95 \%$ \\
\hline \multicolumn{7}{|l|}{ SEXO } \\
\hline Henulne & 215 & 36.7 & 0.75 & $0.80-0.95$ & 0.35 & $0.57-0.98$ \\
\hline Mıјढ & $26 \mathrm{kB}$ & & Refercuicta & & & \\
\hline \multicolumn{7}{|l|}{ HOSPITAL } \\
\hline $\mathrm{Si}$ & 22 & 37,3 & 0,80 & $0,50-1,57$ & 0.8 & as \\
\hline No & 491 & & Refercacia & & & \\
\hline \multicolumn{7}{|l|}{ CONSUILA } \\
\hline c:s & 449 & 79,1 & 0,7 & $0,51-1,0$, & 0,75 & $\mathrm{~ns}$ \\
\hline Donicilio & 64 & & Referencin & & & \\
\hline \multicolumn{7}{|l|}{ POBBLACION } \\
\hline Tatiallit & 432 & & Refercatiata & & & \\
\hline De:plitrandos: & 81 & 42,4 & 1,13 & $0 \times 0.1,56$ & 12 & $\mathrm{~ns}$ \\
\hline \multicolumn{7}{|l|}{ GRUPHORA } \\
\hline $15-17$ & 29 & 19.1 & $0.0 \mathrm{BS}$ & $0.05-0.12$ & 0.04 & $0.01-0,0.05$ \\
\hline 1620 & 161 & 24,2 & 0.1 & $0,0,1 \mathrm{~K} 0,14$ & 0,07 & $0,0.50,(x)$ \\
\hline $2.1-2: 3$ & $30 x$ & & Refierencia & & & \\
\hline$a-2$ & 15 & 21,6 & 0,1 & $0,05-0.20$ & 0.12 & $0,060.21$ \\
\hline \multicolumn{7}{|l|}{ (IRUPVDAD) } \\
\hline 04 & 81 & $4: 3,1$ & $0, \$ 4$ & $(0,6331,40)$ & 1,0 & ns \\
\hline $5-9$ & 41 & 519 & 1,74 & $0,79-2.79$ & 152 & $\mathrm{~ns}$ \\
\hline $10-21$ & 101 & 39 & 0.79 & $0.55-1,15$ & 0.86 & as \\
\hline 2529 & 30 & 25,6 & $(1,4,3$ & $(1,260,31$ & 0.47 & 0. $260,0,87$ \\
\hline $30-49$ & 115 & & Refierencia & & & \\
\hline $50-54$ & 14 & 25 & 0,41 & $0.20-0,83$ & 0.44 & $0.20-0,90$ \\
\hline $55 \mathrm{y}(+)$ & & $4, G$ & 0.81 & $0,57-1,14$ & 0,95 & as \\
\hline \multicolumn{7}{|l|}{ CAMPEONATO } \\
\hline I رئ & 326 & & Reletrencasa & & & \\
\hline Uefin & 187 & 448 & 1,35 & $1,(x,-1,73$ & 1.94 & $1,37-2,32$ \\
\hline \multicolumn{7}{|l|}{ TELEVISION } \\
\hline I'ublica & 312 & 12.4 & 1.35 & $1.05-1.33$ & 0.22 & $0.19-(1.44$ \\
\hline I'nvada & 164 & & Reléremeata & & & \\
\hline Puiblusa I Privada & 37 & 44 & 1,4 & $0 \times 88-2,37$ & 0.4 & ואד, (1) \\
\hline \multicolumn{7}{|l|}{ DIAGNOSTICO } \\
\hline 1 & 5 & 19,2 & 0,35 & $0,110,98$ & 0,41 & $\mathrm{~ns}$ \\
\hline 11:se & 50.4 & & Refierencia & & & \\
\hline VII & 4 & 19 & 0.34 & $0,10-1,09$ & 0,45 & as \\
\hline
\end{tabular}

estos meses se celebran las dos fiestas patronales de la localidad de Tafalla y corresponden a la mayor frecuentación diaria, con 73 episodios/día respectivamente para cada mes. No obstante, otros estudios señalan resultados muy diferentes ${ }^{13}$.

Rev Esp Salud Pública 2003, Vol. 77, N. ${ }^{\circ} 6$ 
Es en invierno cuando más atención a domicilio se produce, motivado posiblemente por las inclemencias climatológicas. Existen diferencias en la demanda entre las diferentes estaciones por grupo de edad. Así, en invierno predomina el grupo de menores de 5 años, de 10 a 14 años en primavera y de 20 a 29 años en verano. Una mayor predisposición a las infecciones respiratorias agudas en los niños menores y una mayor movilidad motivado por las fiestas patronales y vacaciones estivales pueden motivar dicha distribución.

Al igual que en otros trabajos, la mayor afluencia es a primeras horas de la tarde, acaso motivada por la atención directa y sin espera, la jornada laboral de los usuarios y la existencia de urgencias subjetivas para tranquilidad del enfermo, así como la comodidad del usuario ${ }^{14-16}$. Las diferencias observadas en la distribución horaria dejan de ser significativas si no se consideran las horas de consulta de 9 a 15 horas propias de los domingos.

Se observan diferencias en la frecuentación horaria por época estacional. Así de 12 a 14,59 horas es más frecuente en otoño e invierno, mientras que en verano existe más demanda en el horario de 21 a 0,59 horas, a causa posiblemente de la buena climatología. El 50\% del total de las visitas domiciliarias se realiza de 15 a 20,59 horas. La coincidencia entre los avisos domiciliarios y el final de la jornada laboral hace cuestionarse si el horario de los avisos no dependerá en parte de las conveniencias personales ${ }^{17}$. Se ha señalado que «el SNU se convierte en la prolongación vespertina y nocturna del pediatra y medico de cabecera» ${ }^{18}$.

La distribución de los episodios por grupo etario presenta una distribución similar a otro estudio consultado ${ }^{19}$. El hecho de referir la edad del paciente a la edad de consulta del primer episodio de atención, pensamos que no introduce ningún sesgo de clasificación en la distribución por grupo etario. La aten- ción domiciliaria aumenta progresivamente con la edad; el 52,3\% del total corresponde al grupo de 70 a 89 años. La hospitalización de los enfermos es uniforme para todos los grupos etarios, con un pico en el grupo de 20 a 29 años, debido fundamentalmente a traumatismos.

En el estudio de los factores relacionados con la sobreutilización de los servicios sanitarios se ha encontrado un perfil típico del hiperfrecuentador ${ }^{20,21}$. Se observa una mayor frecuentación en los menores de 30 años $(50 \%)$ y fundamentalmente en los menores de 10 años $(22,2 \%)^{20,22}$, donde la cercanía al CS parece ser un factor condicionante. En otro artículo ${ }^{23}$ se describe la relación entre morbilidad y utilización en pediatría pero sus resultados son difíciles de comparar, pues no se han empleado sistemas estandarizados para clasificar las enfermedades. Respecto al total de la población atendida, los porcentajes de ingreso hospitalario se reducen a la mitad, mientras que las consultas a domicilio tienen un aumento superior al doble. Algunos autores han encontrado en la mitad de las personas hiperfrecuentadoras la presencia de malestar psíquico con familias disfuncionales ${ }^{20,21}$, por lo que resultaría útil para detectar problemas de salud mental, frecuentemente ocultos y manifestados por patologías somáticas. Sin duda estos enfermos o sus familiares demuestran un buen conocimiento de las prestaciones sanitarias, bien como consecuencia o bien como causa del uso continuado de dichos servicios. No obstante, otros estudios señalan que la hiperutilización persistente lejos de ser un comportamiento inadecuado es una respuesta apropiada a las mayores necesidades de cuidados sanitarios que presentan estos pacientes ${ }^{23}$.

La fácil accesibilidad de la población ha sido señalada como un factor determinante de la demanda. Esto provoca que los habitantes de la cabecera del centro demanden mayor asistencia que los habitantes de los otros municipios. En nuestro estudio los 
datos confirman estos resultados. Así, el número de episodios vistos respecto al número de TIS es del $68,3 \%$ para Tafalla y del $35,2 \%$ para OP. Incluso se ha evidenciado que en la derivación al hospital el mayor número de derivaciones proviene de municipios que son cabecera de sus respectivos centros $^{24}$, datos no ratificados en nuestro estudio. La mayor distancia al centro de salud, el envejecimiento de la población y la dificultad de traslado son las razones que lo justifican.

La remisión al hospital $(4,8 \%)$ se sitúa en la franja inferior de los resultados obtenidos por otros trabajos publicados ${ }^{19,25}$. La existencia de material diagnóstico básico, la distancia al centro hospitalario ${ }^{26,27}$ y el uso de criterios subjetivos para la evaluación parecen ser determinantes en la remisión al hospital.

Las urgencias domiciliarias suponen el $10,6 \%$ del total, cifras inferiores ${ }^{17,19,24}$ a otros estudios publicados. Las mujeres solicitan mayor atención a domicilio que los hombres posiblemente motivado por una mayor edad ${ }^{17,24}$. Una mayor ancianidad (31,93 años de edad media para los visitados en el centro de salud y 65,9 para los visitados en domicilio) y la existencia de patología concomitante al episodio que motiva la consulta, lo justifican.

Las principales causas atendidas corresponden a los grupos XVII, VIII y XVI, similar a otros estudios ${ }^{28}$ publicados, con el 53,9 \% sobre 9190 diagnósticos. Las infecciones agudas de las vías respiratorias superiores $(8,3 \%)$, las heridas y contusiones $(7,4 \%)$, la amigdalitis aguda $(3,8 \%)$ y el dolor abdominal $(3,1 \%)$ son los procesos mas frecuentes. Estos porcentajes difieren de los resultados de otros trabajos ${ }^{16}$ realizados. Los grupos XVI y XVII representan el $49,5 \%$ del total de ingresos hospitalarios y los grupos $\mathrm{V}$ y XVI representan el $42,4 \%$ de los avisos domiciliarios. La posibilidad de sesgo de clasificación en la codificación de los enfer- mos es débil debido a que únicamente se han contabilizado diagnósticos realizados por los médicos de plantilla.

El alto porcentaje de usuarios desplazados que encontramos en nuestro estudio $(13,3 \%)$ es un factor que distorsiona la organización de urgencias. Se distribuyen a lo largo de todo el año pero más frecuente en abril, julio y fundamentalmente agosto, lo que atribuimos a las vacaciones estivales. El porcentaje de usuarios desplazados hospitalizados sobre el total de desplazados es del 7,94\% y representa el 1,06\% del total de hospitalizaciones.

La asistencia a desplazados se distribuye en todos los tramos horarios (en el grupo de 0 a 5,59 horas 1 de cada 5,5 visitas corresponden a desplazados),en todos los grupos etarios (el 94\% son menores de 34 años) y todos los días de la semana. El 58,13\% de los desplazados son hombres, porcentaje muy similar respecto al total de los episodios vistos. Proceden prácticamente al $100 \%$ de las poblaciones circundantes a la zona de salud y está motivado fundamentalmente por que las guardias en estas poblaciones únicamente son de presencia localizada, lo que según el enfermo provoca retraso en la atención. Excepto para los avisos por accidentes de tráfico en que el comunicante es SOS-Navarra, la población acude al CS o bien pone un aviso domiciliario directamente, sin el filtro que puede suponer SOS, como ocurre mayoritariamente en Navarra. El hecho de no poder contactar directamente con el médico, puede conllevar cierta alarma respecto al tiempo estimado de actuación del médico, que produce como reacción el traslado del enfermo al CS de Tafalla. Otro factor a considerar es que al haber en Tafalla diferentes especialidades médicas a las que acude la población de las áreas cercanas, también para las consultas de urgencias lo consideran como centro de referencia.

Los mismos grupos XVII, VIII Y XVI son los mas frecuentes, comprendiendo el 53,02 
$\%$ del total de diagnósticos para desplazados (sobre un total de 1224 diagnósticos). Así, las razones que motivan las consultas urgentes en los desplazados corresponden al mismo grupo que para el total de episodios vistos.

Se observan diferencias tanto en episodios vistos como en atención domiciliaria y remisión a hospital respecto al médico de cabecera asignado. Estas diferencias parecen deberse mas al propio proceso de creación de los diferentes cupos sanitarios que a característicos del usuario como edad, sexo y patología o bien del propio personal médico. Estos datos no han podido ser contrastados al no encontrar estudios al respecto.

No se ha calificado la urgencia como adecuada/inadecuada, aunque algunos autores señalan que el porcentaje de inadecuación puede llegar hasta el $62 \%{ }^{2}$ con las consiguientes consecuencias de aumento de costes, frustración profesional y atención medica fragmentaria.

La retransmisión de fútbol por televisión se ha asociado a un aumento del $19,8 \%$ en la demanda de urgencias lo que hace discutible que, además de las razones médicas, existen otros factores relacionados no directamente con la enfermedad, que pueden motivar que los pacientes acudan o no a un servicio de urgencias. La retransmisión para el único equipo de primera división de la comunidad se realiza por medio de la televisión privada, de pago, y parece ser el motivo de la mayor relevancia de las retransmisiones por televisión pública o gratuita. En el análisis multivariante se demuestra la aparición del fenómeno de confusión (paradoja de Simpson) para la variable televisión.

Otros dos estudios, únicos del que tenemos constancia, reflejan resultados muy diferentes en la afluencia de demanda si bien referido al ámbito de las urgencias hospitalarias $^{11,29}$. En nuestro estudio, la amplitud del número de partidos de fútbol retransmitidos por televisión, la cercanía de la población y la rapidez en la atención han motivado las diferencias metodológicas ${ }^{11}$ en lo referente al periodo-control respecto a los estudios anteriores.

La alta frecuentación de los servicios de urgencias puede explicarse por la facilidad de acceso y por la expectativa de un servicio asistencial rápido, sin listas de espera. La mayor utilización del servicio por los varones en edad laboral puede ser debido a que sus turnos de trabajo no les permite asistir en horario de mañana ${ }^{30}$ a la consulta.

Algunos resultados deben ser interpretados con cautela, debido a las siguientes limitaciones. En primer lugar, no se ha hecho distinción entre días no festivos y festivos, considerando tales únicamente los domingos, lo que puede generar dificultades en la comparación de resultados. En segundo lugar, es posible un sesgo de clasificación en los desplazados, al no comprobar la veracidad de la información solicitada, subestimando probablemente la cantidad total. Otra limitación deriva de que se asume que la distribución de la demanda en horario de fútbol televisado y de su periodo control es uniforme para todo el periodo de estudio.

Coincidimos con la mayoría de los autores consultados en la importancia de una mayor educación y concienciación sanitaria de la población y de la necesidad de utilización de estos servicios de manera adecuada.

\section{BIBLIOGRAFIA}

1. Organización Mundial de la Salud. Alma-Ata 1997. Atención Primaria de Salud. Ginebra: OMS UNICEF; 1978.

2. Grupo de trabajo de la SAMFYC. Ordenación de las urgencias en Aten Primaria 1992;9: 269-75.

3. Rodríguez Alcántara F, Rodrigo García-Pando C Hernández Colan I, Lozano Quintana A, Herranz Diez I. ; Doctor, vengo de urgencias! Aten Primaria 1998;22:655-60. 
4. INSALUD. La afluencia a urgencias crece a un ritmo constante. Diario Médico, 2-III-1998; 8.

5. Ullman R, Black JA, Boatright NC, Stratman WV Impact a primary care group practice on emergency room utilisation at a community hospital. Med Care $1978 ; 16: 723-9$

6. Millá Santos J. Urgencias médicas: algo mas que una serie televisiva. Med Clín 2001;117: 295-6.

7. Menéndez JM. Gestión de las urgencias. Organización y Gestión de la atención urgente extrahospitalaria. En: Del Llano J, Ortún V, Martín JM, Gené J. Gestión Sanitaria. Innovaciones o desafíos. Barcelona: Masson SA; 1997.p.403-18.

8. Ministerio de Sanidad y Consumo. Urgencias y emergencias sanitarias. Madrid: Subdirección General de Evaluación sanitaria y Tecnología. Ministerio de Sanidad y Consumo; 1991.

9. Fernández Cano G, Martín Carballo G. Urgencias pediátricas atendidas en una consulta de atención primaria (I): análisis de la demanda. Aten Primaria 2000;2:28-36.

10. Trillo Fernández C, García Guerrero J, Oliva García JM, Martínez Diz S, Lillo Moreno C, Muñoz Hernández A. Estudio de los factores que influyen en la hiperutilización del Servicio de Urgencias en nuestro Centro de Salud. Cent Salud 1998;6:298303.

11. Miro O, Sánchez M, Borras A, Millá J. Fútbol, televisión y servicios de urgencias. Med Clín 2000 14:538-9.

12. Clasificaciones de la WONCA en atención primaria: CIPSAP-2-Definida. Barcelona: Editorial Masson; 1988.

13. Alvarez-Tutor E, Alvarez-Tutor J, Ciriza C, Aznal MV, Navas MI. Estudio descriptivo de las urgencias extrahospitalarias producidas durante un año en un centro de salud de Navarra. Perfil de los pacientes que las frecuentan. Cent Salud 2000; p:41-45.

14. Ramos Martínez J. Perfiles epidemiológicos de las urgencias atendidas durante un quinquenio en un centro de salud. Salud Rural 1997:13:87-93.

15. Belzunegui A. Distancia del hospital y utilización de los servicios de urgencia. Actas del X Congreso de la Sociedad Española de Medicina Familiar y Comunitaria. Barcelona; 1990.
16. Inglada Galiana L, García Morras C, Martín Marcos, C, Martín Baeza AM, Jiménez López T, De Paz García M. Evaluación de la demanda de atención urgente en un hospital comarcal y las zonas básica de salud adscritas. Salud Rural 2001; 9: 43-57.

17. Sanchez Montero MB, Vaquero Roncero LM Urgencias domiciliarias: las olvidadas en Atención Primaria. Centro Salud 2000; 8: 425-29.

18. Leon M, Anguera S, Moya E, Agustí E. Estudio cualitativo de la demanda de asistencia domiciliaria de un Servicio Especial de Urgencias. Aten Primaria 1989;6:98-102.

19. Alonso J, Ramos C, López P, García M, Sánchez I, Hernández C, Sanz L. Urgencias médicas en la zona de salud de Ocaña. Un año de demanda. zona de salud de Ocaña.

20. Llorente Alvarez S, López Ruiz T, García Lavandera LJ, Alonso Arias P, Muñoz Baragaño P, Alonso Fernández M. Perfil del hiperfrecuentador de un centro de salud. Aten Primaria 1996; 17:100-107.

21. Fuentes Goñi MC, Alvarez Tutor E, García de Noceda Montoya MD, Fernández Velilla Ruiz de la Torre M, Echarte Esacain E, Abad Vicente J. Características del hiperfrecuentador de las consultas de demanda de medicina general. Aten Primaria 1994; 11: 809-14.

22. Rodriguez Alcántara F, Rodrigo García-Pando C, Hernández Colau I, Lozano Quintana A, Hernández Diez I.; Doctor, vengo de urgencias! Aten Primaria 1998; 10: 655-60.

23. ¿Es necesario que algunos pacientes nos visiten tan a menudo?: Factores asociados con la utilización en pediatría de atención primaria. Gac Sanit 2000;14(3):195-202.

24. Lorenzo-Caceres A, Ama JA, Arcos L, Brezmes JA, Serralle JR, Mateo S et al. Estudio descriptivo de la demanda de urgencias en el medio rural. Rev San Hig Pública 1986; 60: 511-28.

25. Castilla J, Duque G, López M. Estudio epidemiológico de la demanda al servicio de urgencias de un centro de salud rural. Salud Rural 1994; 11: 19-24.

26. Valls Soler J, Segura Monreal L, Viñas M, Avendaño E. Urgencias en Atención Primaria y derivación de pacientes al hospital. Aten Primaria 1990; 7: de pacien.

27. Plans Pedro A, Caylá Buqueras JA, Vidal Marsal A, Plana Pey J, Riera Martí L, Martínez Vuelta I.

Rev Esp Salud Pública 2003, Vol. 77, N. ${ }^{\circ} 6$ 
PERFIL DE LA DEMANDA URGENTE E INFLUENCIA DE FUTBOL TELEVISADO EN UN SERVICIO.

Análisis de las prestaciones realizadas en un servicio de urgencias de asistencia primaria. Gac Sanit $1986 ; 5: 105-9$.

28. Blanco Montagut LE. Informe de la actividad asistencial del punto de atención continuada de Fuentes de Oñoro(Salamanca). Cent Salud 1994; 2:465-70
29. Reich N, Moscati R, Jehle D, Ciotoli M. The impact of a major televised sporting event on emergency department census. J Emerg Med 1994; 12:15-7.

30. Campillos Páez MT, Gómez Díaz E, Campillos Páez MA. Urgencias atendidas en un centro de salud urbano. Cent Salud 1999; 7(9): 552-5. 